\title{
Was ist für die Diagnostik und Therapieverlaufs- kontrolle von chronischem Husten wichtig?
}

\author{
What is Important for Diagnosis and Therapy Follow-up in Chronic Cough?
}

Autoren

Institute
U. Koehler ${ }^{1}$, O. Hildebrandt ${ }^{1}$, U. Walliczek ${ }^{2}$, L. Höhle ${ }^{3}$, A. Weissflog ${ }^{3}$, J. Heselhaus ${ }^{3}$, S. Kerzel ${ }^{4}$, C. Urban ${ }^{4}$, K. Sohrabi ${ }^{5}$, V. Gross ${ }^{5}$

Die Institutsangaben sind am Ende des Beitrags gelistet. eingereicht 10.6.2015 akzeptiert nach Revision 3.7.2015

Bibliografie

DOI http://dx.doi.org/

10.1055/s-0034-1392616

Pneumologie 2015; 69: 588-594

(c) Georg Thieme Verlag KG

Stuttgart · New York

ISSN 0934-8387

Korrespondenzadresse Prof. Dr. med. Ulrich Koehler

Klinik für Innere Medizin SP Pneumologie, Intensiv- und Schlafmedizin

Philipps-Universität

Baldingerstraße 1

35043 Marburg

koehleru@med.uni-marburg.de

\section{Zusammenfassung \\ $\nabla$}

Auf dem Gebiet der Analyse chronischen Hustens sind in den letzten Jahren erhebliche Fortschritte gemacht worden. Bislang erfolgte die Erfassung und Beurteilung von chronischem Husten primär subjektiv anhand von Fragebögen und Tagebüchern. Die Beurteilung von chronischem Husten sowie dessen Therapie sollte zukünftig, insbesondere unter evidenzbasierten Kriterien, einer Kombination aus objektiven und subjektiven Kriterien unterzogen werden. Objektive Kriterien leiten sich dabei aus einer $24 \mathrm{~h}$-Hustenregistrierung und Hustenprovokationsmessung (Hypersensitivität) ab, subjektive aus der Bewertung gut validierter Fragebögen zu Husten und Lebensqualität. Hinsichtlich der 24h-Langzeitregistrierung von Husten sollte primär die Hustenfrequenz erfasst werden. Weitere diagnostische Optionen der Feinanalyse (produktiver, nicht-produktiver Husten) sind gegeben.

\section{Das Symptom Husten \\ $\nabla$}

Husten ist ein physiologischer Reflex, der als Schutzmechanismus der Atemwege dient. Fremdkörper oder -stoffe werden durch den Hustenstoß schnell und effektiv aus dem Atemweg entfernt. Husten dient aber auch als nachgeschalteter Reinigungsmechanismus, wenn das primäre „Clearance- oder Reinigungssystem“ der Bronchien nicht mehr funktioniert. Eine effektive Reinigung der Atemwege setzt weitgehend intakte anatomische und physiologische Verhältnisse voraus. Je stärker die mukoziliäre Clearance aufgrund chronischer Noxen (Inhalationsrauchen) oder in Folge eines akuten Infektes geschädigt ist, desto mehr ist das Bronchialsystem auf einen adäquaten Hustenreflex angewiesen [1]. Ohne Husten würde das Sekret im Bronchialsystem verbleiben. Es gibt vier bedeutsame Clearance-Mechanismen: die Sekretolyse, die Sekretmobilisierung, den Sekrettrans-

\section{Abstract \\ $\nabla$}

Within the last years there has been significant progress in the field of chronic cough. So far, the analysis and evaluation of chronic cough was done mainly on the basis of subjective methods such as manual counts of cough events, questionnaires and diaries. Testing cough hypersensitivity and monitoring $24 \mathrm{~h}$ cough represent objective criteria. Validated questionnaires on cough frequency and quality of life represent the impact of chronic cough. Cough frequency monitoring, the preferred tool to objectively assess cough, should be used as primary end-point in clinical trials. It will also be possible to discriminate between productive and non-productive cough. The relationship with subjective measures of cough is weak. In the future, cough and its therapy should therefore be assessed with a combination of subjective and objective tools.

port sowie die Sekretelimination. Durch Erhöhung des Sekretvolumens (Sekretolytika) und eine Herabsetzung der Viskosität (Mucolytika) soll die Clearance des Bronchialsystems verbessert werden. Bislang gibt es nur wenige methodisch einwandfreie Studien zur Bewertung der Wirksamkeit verschiedener Sekretolytika und auch Hustenstiller.

Husten wird in produktiven und nicht-produktiven Husten unterteilt, die Übergänge sind jedoch zumeist fließend. Von großer Relevanz ist die Dauer des Auftretens von Husten. Nach heutigen Kriterien wird chronischer Husten bei Erwachsenen als solcher definiert, wenn er über mindestens 8 Wochen anhält und seine Ursache nicht durch Anamnese, körperliche Untersuchung, Lungenfunktion und eine Röntgenaufnahme des Thorax erklärbar ist. Eine selbst- oder fremdinduzierte Schadstoffbelastung (Rauchen) kann zu chronischem Husten führen. Bei Patienten, bei 




Abb.1 Bei der Hustenaktion können die Inspirations-, die Kompressions- sowie die Expulsionsphase unterschieden werden. Mit der Öffnung der Glottis geht ein heftiger Atemstoß (erstes Hustenereignis) einher, danach folgen eine Abnahme des Atemflusses sowie ein zweites Hustengeräusch durch Glottisschluss (Grafik verändert in Anlehnung an Korpáš et al., 1996, [13]).

denen sich ursächlich „keine“ pulmonale Grunderkrankung nachweisen lässt, sollte differenzialdiagnostisch an eine gastroösophageale Refluxkrankheit (GERD), ein upper airway cough Syndrom (postnasal drip Syndrom, chronische Rhinosinupathie), ein cough variant Asthma, einen medikamentös induzierten Husten sowie eine obstruktive Schlafapnoe (OSA) gedacht werden [2 -7]. Als idiopathischer Husten wurde die Krankheitsentität bezeichnet, bei der keine Ursache zu finden war. Um den Husten adäquat therapieren zu können, ist die Abklärung der Ursache zwangsläufig notwendig. Chronischer Husten ist mit einer deutlichen Beeinträchtigung der physischen Leistungsfähigkeit sowie der Lebensqualität verbunden $[8,9]$. Dabei sind soziale Isolierung, Schlaffragmentation und bei Frauen Stressinkontinenz nur beispielhaft zu nennen.

\section{Der Hustenreflex}

Der Hustenreiz wird im Wesentlichen durch mechanische und chemische Reizung von Rezeptoren im Bereich von Larynx, Hypopharynx, Trachea und großen Bronchien hervorgerufen [10]. Es werden Hustenrezeptoren vom Aס-Faser- und C-FaserTyp unterschieden. Während es sich bei den Aठ-Faser-Rezeptoren um Mechanorezeptoren handelt, sind die C-Faser-Rezeptoren primär auf chemische Reize reagierend. Am dichtesten sind die Hustenrezeptoren im Larynx und im tracheobronchialen Baum vorhanden. Die tieferen Atemwege sind weniger mechanosensitiv als die oberen, dafür ist deren Chemosensitivität höher. In der Lungenperipherie, im Niveau von Bronchiolen und Alveolen, ist keine zu Husten führende mechanische/chemische Irritation mehr nachweisbar.

In der Lunge selbst sind bronchopulmonale Dehnungsrezeptoren, Irritant-Rezeptoren und J-Rezeptoren nachweisbar. Schnelladaptierende bronchiale Irritant-Rezeptoren findet man unter der Schleimhaut von der Trachea bis zu den Bronchiolen. Husten kann jedoch auch über die alveolaren juxtakapillären Rezeptoren (J-Rezeptoren), die sich in den alveolaren Kapillarwänden befinden, getriggert werden. J-Rezeptoren werden stimuliert, wenn die Menge interstitieller Flüssigkeit in der Lunge zunimmt, also bei dekompensierter Herzinsuffizienz, Lungenödem oder Lungenembolie. Alle in der Lunge befindlichen Nervenfasern bilden ein axonales Netzwerk, welches in direkter Verbindung mit den parasympathischen Ganglien steht. Auch die intrapulmonalen Dehnungsrezeptoren, die pleuralen Schmerzfaserrezeptoren so- wie taktile Rezeptoren im äußeren Gehörgang sind im Hustenreflexbogen verschaltet.

Der am Rezeptor aufgenommene Reiz wird über einen nervalen Reflexbogen zum Hustenzentrum geleitet und dort verarbeitet. Vom Hustenzentrum aus werden über efferente Nervenfasern das Diaphragma, die Interkostalmuskulatur sowie der Larynx stimuliert, welche daraufhin einen Hustenstoß auslösen. Der Einfluss weiterer Hirnareale zeigt sich dadurch, dass Husten nicht nur reflexartig, sondern auch willkürlich ausgelöst und andererseits Husten unterdrückt werden kann.

Aktuellere Auffassungen zur Ätiologie chronischen Hustens gehen von dem gemeinsamen Konzept der Hypersensitivität aus. Der Oberbegriff wird als „cough hypersensitivity syndrome“ bezeichnet. Pathophysiologisch wird gemutmaßt, dass eine vermutlich inflammatorisch getriggerte Sensibilisierung afferenter Nervenfasern zum Auftreten von chronischem Husten führt [11, 12]. Dabei ist nicht entscheidend, wodurch die Inflammationsreaktion an den peripheren Nervenfasern oder den Rezeptoren induziert wird. Das kann sowohl ein Folgezustand nach viralem oder bakteriellem Infekt oder saurem bzw. basischem Reflux sein. Mit diesem neuen Konzept wird sozusagen eine pathophysiologische Gemeinsamkeit geschaffen, unter deren Schirm sich dann die verschiedenen Phänotypen upper airway cough Syndrom, cough variant Asthma, gastroösophagealer Refluxhusten, medikamentös induzierter Husten und Husten bei obstruktiver Schlafapnoe subsumieren lassen.

\section{Ablauf einer Hustenaktion}

$\nabla$

Unabhängig davon, ob ein Hustenstoß willkürlich oder unwillkürlich herbeigeführt wird, können folgende drei Phasen unterschieden werden: Inspirationsphase, Kompressionsphase mit Glottisverschluss und Expulsionsphase ( Abb.1). Begonnen wird ein Hustenstoß mit der Inspirationsphase, die wichtig ist für die Intensität und Effektivität des Hustenstoßes. Nach der tiefen Inspiration kommt es zum Glottisverschluss bei gleichzeitiger maximaler Kontraktion der exspiratorischen Atemmuskeln, wodurch ein hoher intrathorakaler Druck aufgebaut wird. Durch das aktive Öffnen der Glottis wird dieser schlagartig reduziert. Mit der raschen Glottisöffnung kommt es zu einer explosionsartigen Exspiration, bei der sehr hohe Flussgeschwindigkeiten erreicht werden können. Mit der forcierten Exspiration wird die eigentliche Aufgabe des Hustens erfüllt, und Fremdkörper werden aus 

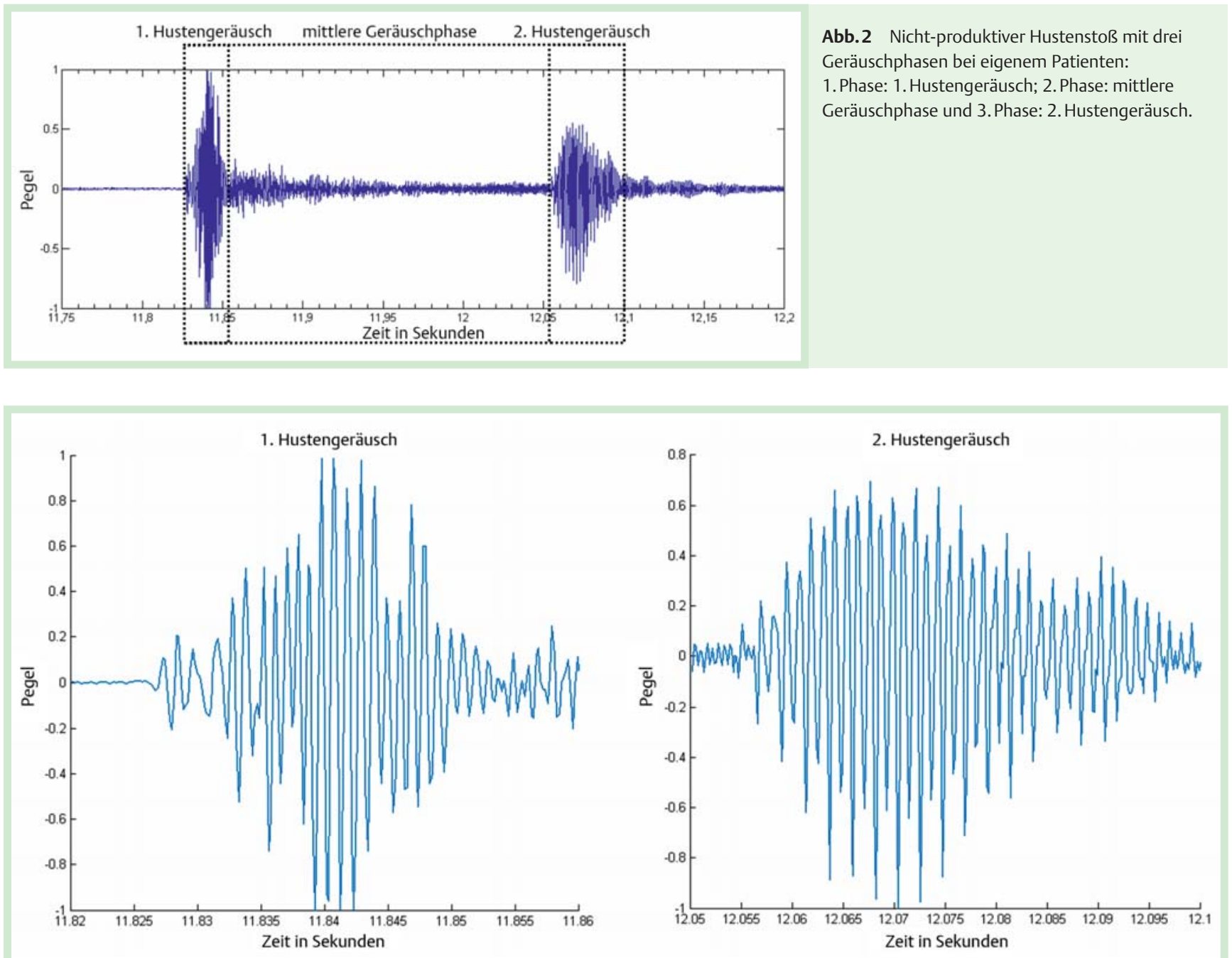

Abb.3 Das erste Hustengeräusch (links) mit rauschähnlichem, das zweite (rechts) mit stimmhaftem Signal bei hoher zeitlicher Auflösung; Messung bei eigenem Patienten.

den Atemwegen herausbefördert. Durch die hohen intrathorakalen Drucke in der Kompressionsphase kommt es zu einer dynamischen Einengung der Querschnitte von Bronchien und Trachea. Bei einem einzelnen Hustenstoß, der mit erheblichen Rückwirkungen auf die Hämodynamik einhergehen kann, dauert die Kompressionsphase nur etwa 250 ms.

\section{Charakteristisches Hustengeräusch}

$\nabla$

Yanagihara und Mitarbeiter [14] haben mittels Hochgeschwindigkeitsaufnahmen das Muster eines Hustengeräusches aufgezeichnet. Durch gleichzeitige Aufnahmen von Flussrate, Glottisfunktion und Hustengeräusch konnte ein zeitlicher Zusammenhang zwischen den einzelnen Abläufen hergestellt werden ( $\bullet$ Abb. 1). Nach der Inspirations- und Kompressionsphase entsteht mit dem Öffnen der Glottis, also dem Beginn der Expulsionsphase, das erste Hustengeräusch. Das typische Geräusch beim Husten entsteht aufgrund von Turbulenzen der ausströmenden Atemluft, Vibrationen des Gewebes und Bewegung von Flüssigkeit durch die Atemwege. Die Lautstärke des Geräusches ist dabei abhängig von der Atemflussgeschwindigkeit, die beim Hustenstoß erreicht wird, der Dichte der Atemluft und der Di- mension der Atemwege. Die mittlere Geräuschphase (Phase zwischen erstem und zweitem Hustenereignis) wird durch einen kontinuierlichen Atemfluss bei weiterhin geöffneter Glottis hervorgerufen. Mithilfe dieser Phase lässt sich am ehesten eine Differenzierung von nicht-produktivem und produktivem Husten vornehmen ( $\bullet$ Abb.2; $\triangle$ Abb.5a,b). Zum Ende der Expulsionsphase schließt sich die Glottis und bewirkt durch die lokal erhöhte Flussgeschwindigkeit wiederum ein Geräusch. Dieses zweite Hustengeräusch tritt allerdings nicht obligatorisch bei jedem Hustenstoß auf. Während des zweiten Hustengeräusches zeigen sich in der Atemflusskurve Schwingungen, die während des ersten Hustengeräusches nicht vorhanden sind ( $\bullet$ Abb.3). Dieser Unterschied belegt anschaulich die verschiedenen Ursprünge der beiden lauten Hustengeräusche. Während des ersten Hustengeräusches zeigt sich ein unregelmäßiges, rauschähnliches Signal. Im Gegensatz dazu hat das zweite Hustengeräusch oft auch regelmäßige, periodische Anteile, welche deswegen auch als stimmhaft bezeichnet werden. 


\section{Quantifizierung des Hustens}

Die diagnostische Einordnung von chronischem Husten erweist sich weiterhin als schwierig. In den letzten Jahren sind jedoch eindeutige Fortschritte gemacht worden, sowohl was die subjektiven als auch objektiven Methoden und Kriterien der Diagnostik betrifft. Die Etablierung gut validierter Fragebögen zur Beurteilung von chronischem Husten und hustenbedingter Lebensqualität sowie die Weiterentwicklung von Systemen zur Langzeitregistrierung von Hustenereignissen eröffnen neue Perspektiven. Aufgrund der häufig nur partiellen Übereinstimmung der Ergebnisse bislang angewandter diagnostischer Verfahren ist die kombinierte Nutzung und Bewertung subjektiver und objektiver Methoden sinnvoll. Die subjektive Beurteilung von chronischem Husten sowie die Bestimmung der Hustenreflexsensitivität erweisen sich beispielsweise als schlechte Surrogatparameter für die objektiv bestimmte Hustenrate bei Patienten mit Asthma bronchiale [15]. Objektive Verfahren sind die Messung der Sensitivität des Hustenreflexes sowie die Langzeitregistrierung von Husten, subjektive die Fragebögen zur Beurteilung von Husten und Lebensqualität.

Die Einschätzung von Häufigkeit und Qualität von Husten durch die Betroffenen selbst oder bei Kindern durch ihre Eltern setzt voraus, dass Husten/Räuspern auch hinreichend genau differenziert und quantitativ erfasst werden kann. Die subjektive Beurteilung von Husten stimmt dabei oft nicht mit den objektiven Messdaten überein. Zu bedenken ist zudem, dass nächtlich auftretender Husten bei Erwachsenen bei geringerer objektiver Ausprägung deutlich schwerwiegender beurteilt wird als derjenige, der tagsüber auftritt. Insbesondere unter den dargestellten Problemen der Fehlbeurteilung von Symptomen sowie dem Aspekt einer evidenzbasierten Therapie ist der objektive diagnostische Nachweis von Hustenereignissen sowie die Verlaufskontrolle von Husten unter einer medikamentösen (antitussiven, sekretolytischen) Therapie zu fordern. Der „Leicester Hustenfragebogen“ sowie der „Fragebogen zur hustenspezifischen Lebensqualitätsbeurteilung" sind die am besten validierten und am häufigsten verwendeten Fragebögen zur Beurteilung chronischen Hustens bei Erwachsenen [16-20]. Die „visuelle Analogskala“ dient zur Beurteilung des Hustenschweregrades. Mithilfe der Graduierung „kein Husten“ bis „schwergradiger Husten“, dargestellt anhand einer $100 \mathrm{~mm}$-Skalierung, soll der Patient sich selbst beurteilen. Bei Patienten mit chronischem Husten ist die Sensitivität des Hustenreflexes im Vergleich zu Normalpersonen gegenüber Capsaicin erhöht [21]. Die Patienten husten bereits bei deutlich geringeren Capsaicin-Konzentrationen als Normalpersonen. Eine einmalige Testung ist jedoch nur bedingt aussagefähig, zumal sich auch bei Gesunden niedrigere Hustenschwellen nachweisen lassen. Eine Provokationstestung mit Capsaicin kann zudem nur selten einen richtungsweisenden Beitrag zur Klärung der Diagnose (Phänotyp) von chronischem Husten leisten. Die endgültige Diagnose bemisst sich in vielen Fällen erst anhand des therapeutischen Erfolgs.

- Abb. 4 stellt die diagnostischen Messinstrumente bei chronischem Husten im Überblick dar. So gibt es derzeit zwei objektive und mehrere subjektive Methoden zur Beurteilung von Husten. Die Bewertung von chronischem Husten sollte demzufolge idealerweise aus einer Kombination der Ergebnisse subjektiver und objektiver Kriterien bestehen. Nicht nur die Beurteilung von Häufigkeit und Schweregrad des Hustens im Tagesverlauf ist sinnvoll, sondern auch die der Beeinträchtigung der Lebensqualität des Patienten und die Differenzierung von produktiven und nicht-produktiven Hustenereignissen.

\section{Akustische Langzeitregistrierung von Husten $\nabla$}

Die Aufnahme von Hustengeräuschen mithilfe von Mikrofonen und digitalen Speichersystemen ermöglicht eine wiederholbare, objektive Beurteilung und Differenzierung des Hustens. Für die Aufnahme von Hustenereignissen stehen verschiedene Sensoren zur Verfügung. Neben der Geräuschaufnahme, die mittels Freifeld-Mikrofon, luftgekoppeltem Mikrofon oder Kontaktmikrofon durchgeführt werden kann, ist außerdem die Aufzeichnung der Thoraxbewegung durch das Hustenereignis möglich. Dafür werden Elektromyografie, Accelerometer und Induktionsplethysmografie genutzt. Zusätzliche Sensoren bringen keine nennenswerten Verbesserungen der Ergebnisse, allenfalls die Objektivierung von Artefakten wie bspw. Sprache und Lachen kann mit zusätzlichen Sensoren verringert werden. Die aktuell zur Verfügung stehenden ambulanten Hustenaufzeichnungssysteme (z. B. Leicester Cough Monitor; Vitalojak; LEOSound) benutzen unter anderem die Hustenrate als primären Endpunkt [18,22 - 24]. Die Erhebung objektiver Daten ist unter evidenzbasierten Kriterien sinnvoll. Dies setzt natürlich voraus, dass die uns zur Verfügung stehenden Messinstrumente die Daten zuverlässig aufzeichnen und bewerten. Das System LEOSound der Firma Heinen und Löwenstein ist zur automatischen Erkennung von Hustenepochen gut geeignet. Im Rahmen einer Validierungsstudie zum LEOSound bei Kindern im Alter von 1 bis 16 Jahren konnte eine Sensitivität von 93\% und eine Spezifität von 99\% für die Erkennung von Husten gezeigt werden [25]. Für die Erkennung von Wheezing lag die Sensitivität bei $98 \%$ und die Spezifität bei 96\%. Die Ergebnisse bei Erwach-

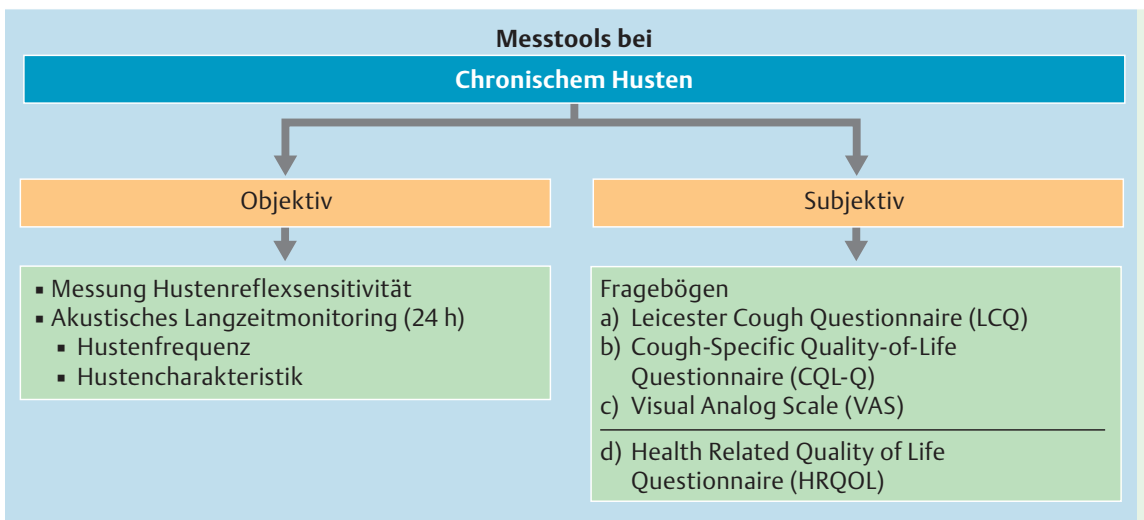

Abb.4 Subjektive und objektive Kriterien zur Beurteilung von chronischem Husten. 
senen zeigen ähnlich gute Werte für Sensitivität und Spezifität (eigene noch unveröffentlichte Daten).

Ambulante Rekorder zur Langzeitregistrierung der Atemwegsgeräusche eröffnen neue diagnostische Optionen. Die akustische Aufzeichnung von Husten über 24 Stunden wird im englischsprachigen Raum bereits als diagnostischer Goldstandard angesehen und gefordert $[17,18,20]$. Was ist messtechnisch von einem ambulanten Hustenmonitoring-System zu fordern?

1. 24h-Messung

2. Sichere Erkennbarkeit von Husten in Abgrenzung zu

3. Sprach- und Störgeräuschen (hohe Spezifizität und Sensitivität der Methode)

4. Erfassung von Husten pro Zeiteinheit

5. Registrierung von Hustenstößen pro Hustenattacke

6. Differenzierung nicht-produktiver und produktiver Hustenereignisse

Die Ausprägung des Hustenschweregrades ist bedeutsam für die Beurteilung der Therapieeffizienz. Husten sollte unter verschiedenen Aspekten beurteilt werden: Häufigkeit des Symptoms, Schweregrad des Symptoms, zeitliches Auftreten des Symptoms sowie Beeinträchtigung der Lebensqualität durch das Symptom. Unter dem Aspekt des Nachweises einer Kausalität von gastroösophagealem Reflux und chronischem Husten ist die synchrone Aufzeichnung von ph-Metrie/Impedanzmessung-ph-Metrie und Hustenmonitoring sinnvoll. Durch eine synchrone Aufzeichnung der Messparameter könnte geklärt werden, ob Refluxereignisse dem Husten oder Husten den Refluxereignissen zeitlich vorausgehen. Ebenso könnte evaluiert werden, in welchem zeitlichen Intervall Husten oder Wheezing einem Refluxereignis folgen. Untersuchungen von Smith et al. [26] sowie Kuntsch et al. [27] konnten anhand der Kombination von akustischer Langzeitregistrierung und ph-Metrie bereits zeigen, dass zeitbezogene Zusammenhänge von Husten und Reflux darstellbar sind. Die Ergebnisse sind jedoch aufgrund kleiner Studienfallzahlen nur bedingt aussagefähig.

\section{Hustenhäufigkeit bei Gesunden und Patienten \\ $\nabla$}

Yousaf et al. [28] führten mittels des Leicester Cough Monitors eine Analyse der Hustenhäufigkeit bei respiratorisch Gesunden sowie bei Patienten mit unterschiedlichen Lungenerkrankungen über 24 Stunden durch. Die Aufzeichnung ergab bei den 44 gesunden Kontrollen im Mittel 18,6 Hustenereignisse, Frauen hatten mehr Husten als Männer (29,5 versus 8,3). Tagsüber (08.00 h bis $22.00 \mathrm{~h}$ ) waren mehr Hustenereignisse als nachts auffällig. Von den 78 Patienten hatten 34 einen unklaren chronischen Husten, 4 Patienten eine ACE-Hemmer-Therapie, 8 ein cough variant Asthma, 9 eine COPD und ein Asthma bronchiale, 5 Patienten eine akute Bronchitis sowie 3 eine interstitielle Lungenerkrankung. 6 Patienten waren gesunde Raucher. In der Gesamtgruppe der Patienten betrug die Hustenrate innerhalb der $24 \mathrm{~h}$ im Mittel 275 Ereignisse. Frauen hatten wiederum mehr Husten als Männer (381 versus 198). Die Hustenhäufigkeit innerhalb der 24 Stunden betrug bei den Patienten mit unklarem chronischem Husten 477, bei denen mit einem cough variant Asthma oder einer interstitiellen Lungenerkrankung 321 bzw. 274 Ereignisse. Interessanterweise fanden sich die Hustenereignisse bei allen Lungenerkrankungen nahezu gleich verteilt über den 24 Stunden-Zyklus. Bei der Validierungsstudie des Leicester Hustenmonitors fanden sich bei 50 Patienten mit chronischem Husten in der 24-stündigen Registrierung ebenfalls signifikant mehr
Hustenereignisse tagsüber als nachts (insgesamt 19/h; tagsüber 25/h; nachts $10 / \mathrm{h}$ )

\section{Husten und Schlaf}

\section{$\nabla$}

Im Schlaf husten Gesunde nicht oder nur selten [9,29]. Untersuchungen zur circadianen Verteilung der Hustenereignisse belegen bei den klassischen chronischen Lungenerkrankungen wie Asthma bronchiale, COPD, interstitielle Lungenerkrankung und Pneumonie eine Reduktion von Husten im Schlaf [9,30,31]. Erklärbar ist dies am ehesten dadurch, dass nachts weniger allergene und auch nichtallergene Stimuli auf den Patienten einwirken. Außerdem sind im Schlaf die Mechano- und Chemorezeptorsensitivität sowie die übergeordneten Einflüsse des Zentralen Nervensystems vermindert. Erkrankungsentitäten wie die gastroösophageale Refluxerkrankung, das upper airway cough Syndrom (postnasal drip Syndrom) sowie das nächtliche Asthma bronchiale und die OSA sollten hinsichtlich des zeitlichen Auftretens von Husten insbesondere im Schlaf genauer analysiert werden. Erste eigene Untersuchungen konnten zeigen, dass die liegende Position im Schlaf das Auftreten von gastroösophagealen und nasopharyngealen Refluxereignissen begünstigen kann $[6,27,32]$. Bei einem Patienten mit chronischer Sinusitis konnten wir in der Langzeitregistrierung der Atemgeräusche ausschließlich im Schlaf auftretende Episoden mit ausgeprägtem Wheezing und Husten nachweisen [32]. Bei der obstruktiven Schlafapnoe kann es, vermutlich durch die apnoeassoziierten intrathorakalen Druckschwankungen bedingt, zu Refluxereignissen und Husten kommen $[3,4,5]$. Eine diesbezügliche Kausalität konnte jedoch bislang nicht bewiesen werden. Interessanterweise erweist sich die CPAP-Therapie als effektive Behandlungsmaßnahme nicht nur der OSA, sondern auch des chronischen Hustens [33,34]. Dieser Therapieerfolg legt den Verdacht nahe, dass ein OSA-assoziierter Pathomechanismus den chronischen Husten bedingt. Der Reflux erscheint dabei durchaus als pathogenetische Erklärung sinnvoll. Es sind aber auch andere Mechanismen vorstellbar, beispielsweise die Austrockung sowie die Inflammation der Schleimhaut durch ein chronisches Vibrationstrauma von Pharynx und Larynx durch Schnarchen bzw. den Atemwegskollaps. Dass der Schlaf hinsichtlich seiner Qualität und Erholungsfunktion durch Hustenereignisse gestört werden kann, erscheint offensichtlich [9]. Bei Patienten mit chronischem Husten psychogenen Ursprungs wird dieser, diagnostisch richtungsweisend, fast ausschließlich in der Tagphase gefunden. Im Schlaf kommt es zu einer deutlichen Abnahme bzw. einem Nichtauftreten von Husten.

\section{Klassifikation von ,produktivem“ und „nicht-produktivem" Husten \\ $\nabla$}

Für die Zuordnung von Hustenereignissen zu den beiden Charakteristika „produktiv“ und „nicht-produktiv“ müssen spezifische Muster aus dem Hustengeräuschsignal extrahiert werden. Verschiedenen Studien nach zu urteilen, liegen die meisten Informationen in der „mittleren Geräuschphase“ des Hustens verborgen ( Abb.5a,b). Demnach weist die zweite Phase bei produktivem Husten einen durchgehend höheren Lautstärkepegel auf und dauert signifikant länger als die bei trockenem Husten. Dies spiegelt sich auch im Verhältnis der zeitlichen Länge der ersten Phase zur Gesamtzeit des Hustenereignisses wider. Die Differenzierung 


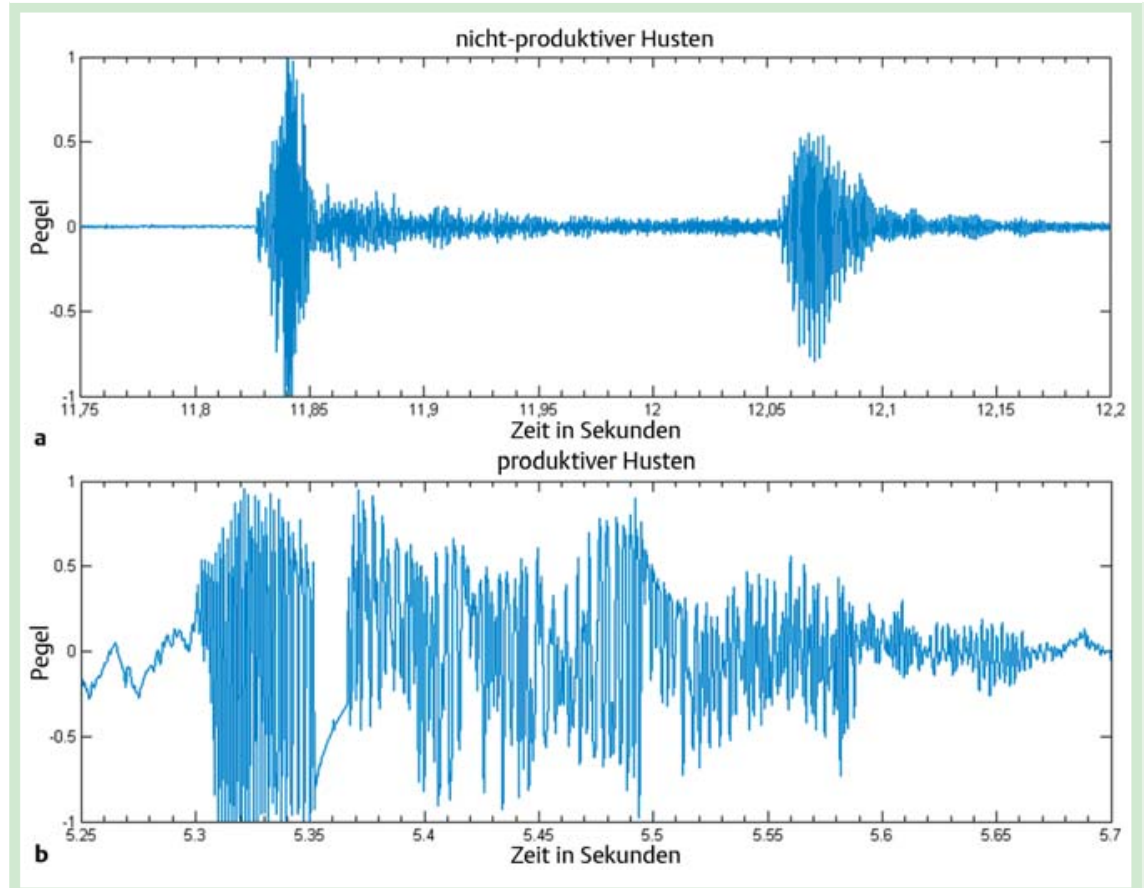

Abb.5 Einzelner Hustenstoß: nicht-produktiver (a) und produktiver Husten (b).

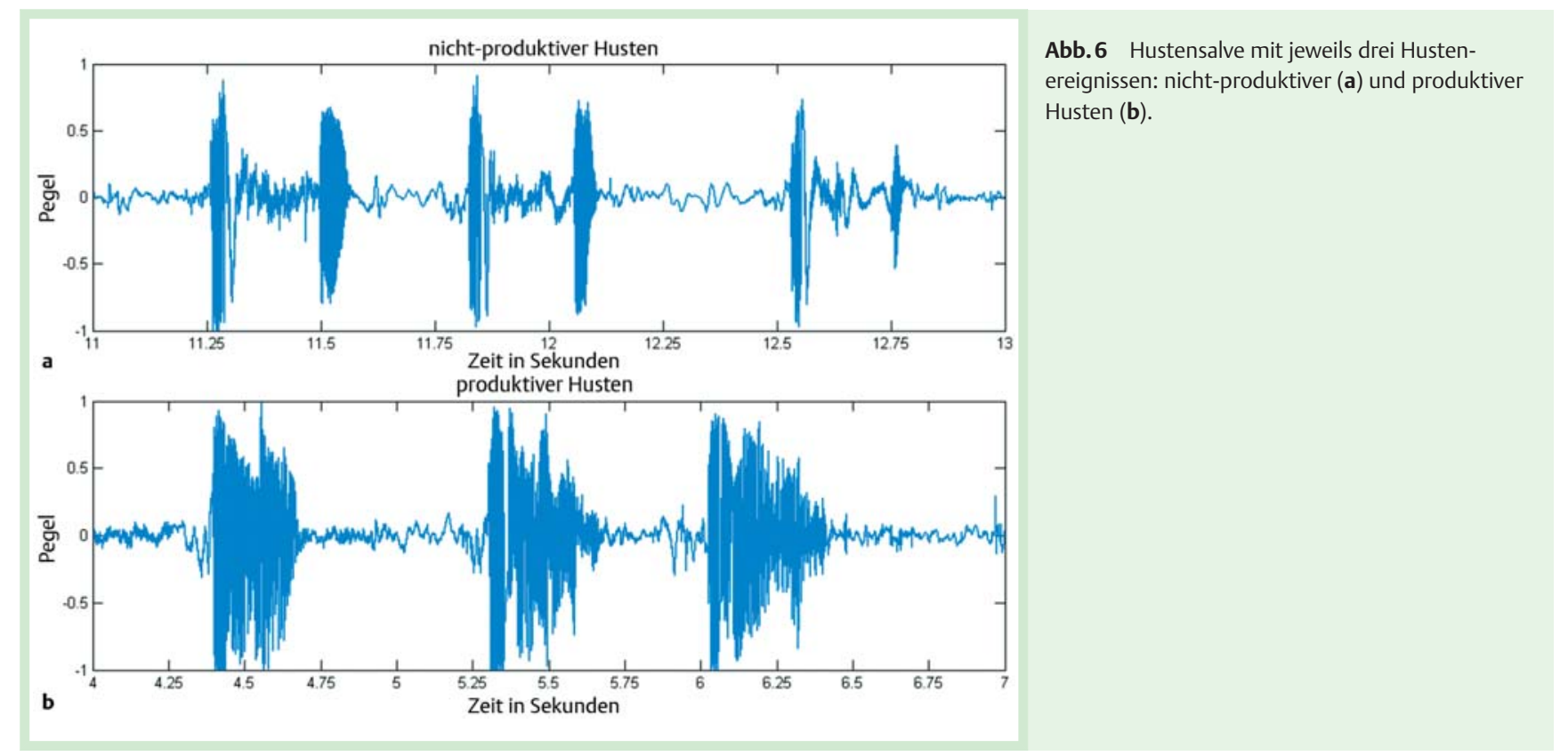

der Hustenereignisse ist insbesondere auch unter dem Aspekt der therapeutischen Konsequenz „antitussiv oder sekretomotorisch" wichtig.

Auf den Abb.6a u. b sind zwei Hustensalven mit jeweils drei Hustenereignissen zu erkennen. Zwischen a und b imponiert die Unterschiedlichkeit der mittleren Geräuschphase. Während es sich bei a) um nicht-produktiven Husten handelt, liegt bei b) produktiver Husten vor. Die Charakteristik und das circadiane Auftreten von Husten (produktiv/nicht-produktiv/mehr Husten im Schlaf als im Wachzustand) können derzeit für keine der aufgeführten Krankheitsentitäten bei chronischem Husten als hinreichend sensibles oder spezifisches Instrument angesehen werden. Allerdings fehlen Daten aus ausreichend großen Studien mit homogenen Einschlusskriterien. Aus den Hustenereignissen allein eine Diagnose ableiten zu wollen, ist zumindest bislang nicht möglich.

\section{Zusammenfassung \\ $\nabla$}

Die Diagnostik und Therapie von chronischem Husten hat in den letzten Jahren Fortschritte gemacht. Die 24-stündige akustische Langzeitregistrierung von Husten stellt ein wichtiges diagnostisches Verfahren dar. Erstmals ist die Möglichkeit gegeben, Hustenereignisse objektiv zu erfassen und zu analysieren. Es besteht dabei auch die Option der Differenzierung von produktivem und nicht-produktivem Husten. Die Bewertung einer medikamentösen Hustentherapie sollte zukünftig - unter evidenzbasierten 
Kriterien - einer Kombination aus objektiven und subjektiven Kriterien unterzogen werden. Objektive Kriterien leiten sich dabei aus der 24h-Hustenregistrierung sowie der Messung der Hustenreflexsensitivität ab, subjektive aus der Erhebung der Daten zur Einschätzung und Beurteilung von Hustenschweregrad und Beeinträchtigung der Lebensqualität mittels gut validierter Fragebögen. Perspektivisch können mithilfe der Kombination von Verfahren (z.B. Impedanz-ph-Metrie und 24h-Hustenmessung) zeitliche Zusammenhänge zwischen chronischem Husten und Erkrankungsentitäten wie GERD, obstruktiver Schlafapnoe und upper airway cough Syndrom nachgewiesen werden.

\section{Interessenkonflikt}

$\nabla$

U. Koehler: Heinen und Löwenstein, IfM, GSK, Resmed, UCB Pharma, Boehringer Ingelheim

V. Groß: Heinen und Löwenstein

O. Hildebrandt, U. Walliczek, L. Höhle, A. Weissflog, J. Heselhaus, S. Kerzel, C. Urban und K. Sohrabi geben an, dass kein Interessenkonflikt besteht.

\section{Institute}

${ }^{1}$ Klinik für Innere Medizin, SP Pneumologie, Intensiv- und Schlafmedizin, Philipps-Universität, Marburg

${ }^{2}$ Klinik für HNO, Philipps-Universität, Marburg

3 Thora Tech GmbH, Gießen

${ }^{4}$ Klinik für Pädiatrische Pneumologie und Allergologie, Universitäts-Kinder-

klinik Ostbayern am KUNO-Standort St. Hedwig, Regensburg

Fachbereich GES, Technische Hochschule Mittelhessen, Gießen

\section{Literatur}

1 Köhler D. Physiology and Pathophysiology of Cough. Pneumologie 2008; 62 (Suppl. 01): 14-17

2 Greenberg H, Cohen RI. Nocturnal asthma. Curr Opin Pulm Med 2012; 18: $57-62$

3 Chan KKY, Ing AJ, Laks L et al. Chronic cough in patients with sleep-disordered breathing. Eur Respir J 2010; 35: 368-372

4 Decalmer S, Stovold R, Houghton LA et al. Chronic cough: relationship between microaspiration, gastroesophageal reflux, and cough frequency. Chest 2012; 142: 958-964

5 Emilsson ÖI, Bengtsson A, Franklin KA et al. Nocturnal gastro-oesophageal reflux, asthma and symptoms of OSA: a longitudinal, general population study. Eur Respir J 2013: 1347 - 1354

6 Dette F, Sohrabi K, Koch B et al. Overnight documentation of respiratory sounds in patients with chronic sinusitis. Med Klin 2010; 105: 695697

7 Palombini BC, Villanova CAC, Araujo E et al. A pathogenetic triad in chronic cough - Asthma, postnasal drip syndrome, and gastrooesophageal reflux disease. Chest 1999; 116: 279-284

8 Brignall K, Jayaraman B, Birring SS. Quality of Life and Psychosocial Aspects of Cough. Lung 2008; 186: $55-58$

9 Lee KK, Birring SS. Cough and sleep. Lung 2010; 188: 91 -94

10 Widdicombe J. Neurophysiology of the cough reflex. Eur Resp J 1995; 8: $1193-1202$

11 Morice AH, Millqvist E, Belvisi MG et al. Expert opinion on the cough hypersensitivity syndrome in respiratory medicine. Eur Respir J 2014; 44: $1132-1148$

12 Morice AH, Jakes AD, Faruqi $S$ et al. A worldwide survey of chronic cough: a manifestation of enhanced somatosensory response. Eur Respir J 2014; 44: 1149-1155
13 Korpáš J, Sadloňová J, Vrabec M. Analysis of the Cough Sound: an Overview. Pulmonary Pharmacology 1996; 9: 261 -268

14 Yanagihara $N$, von Leden $H$, Werner-Kukuk E. The Physical Parameters of Cough: The Larynx in a Normal Single Cough. Acta oto-laryngologica 1966; 61: 495-510

15 Marsden PA, Smith JS, Kelsall AA et al. A comparison of objective and subjective measures of cough in asthma. J Allergy Clin Immunol 2008; 122: $903-907$

16 Birring SS, Prudon B, Carr AJ et al. Development of a symptomatic specific health status measure for patients with chronic cough: Leicester Cough Questionnaire (LCQ). Thorax 2003; 58: 339-343

17 Birring SS, Kavanagh J, Lai K et al. Adult and paediatric cough guidelines: Ready for an overhaul? Pulmonary Pharmacology \& Therapeutics 2015: $1-8$

18 Boulet LP, Coeytaux RR, McCrory DC et al. Tools for Assessing Outcomes in Studies of Chronic Cough. CHEST Guideline and Expert Panel report. Chest 2015; 147: 804-814

19 Kardos P, Berck H, Fuchs KH et al. Guidelines of the German Respiratory Society for Diagnosis and Treatment of Adults Suffering from Acute or Chronic Cough. Pneumologie 2010; 64: 701 -711

20 Morice AH, Fontana GA, Belvisi MG et al. ERS guidelines on the assessment of cough. Eur Respir J 2007; 29: 1256-1276

21 Pullerits T, Ternesten-Hasseus E, Johansson EL et al. Capsaicin cough threshold test in diagnostics. Respir Med 2014; 108: 1371-1376

22 Birring SS, Fleming T, Matos S et al. The Leicester Cough Monitor: preliminary validation of an automated cough detection system in chronic cough. Eur Respir J 2008; 31: 1013 - 1018

23 Koehler $U$, Brandenburg $U$, Weissflog $A$ et al. LEOSound, an innovative procedure for acoustic long-term monitoring of asthma symptoms (wheezing and coughing) in children and adults. Pneumologie 2014; 86: $193-198$

24 Spinou A, Birring SS. An update on measurements and monitoring of cough: what are the important study endpoints? J Thorac Dis 2014; 6: $728-734$

25 Gross V, Scholtes $M$, Sohrabi K et al. Validation of the LEOSound ${ }^{\circledR}$-Monitor for standardized detection of cough and wheezing in children. Accepted for World-Sleep-Conference 2015 Istanbul; in press

26 Smith JA, Decalmer S, Kelsall AA et al. Acoustic Cough - Reflux Associations in Chronic Cough: Potential Triggers and Mechanisms. Gastroenterology 2010; 139: 754- 762

27 Kunsch S, Gross V, Neesse A et al. Combined lung-sound and reflux-monitoring: a pilot study of a novel approach to detect nocturnal respiratory symptoms in gastro-oesophageal reflux disease. Aliment Pharmacol Ther 2011; 33: $592-600$

28 Yousaf $N$, Monteiro $W$, Matos $S$ et al. Cough frequency in health and disease. Eur Respir J 2013; 41: 241 - 243

29 Janson C, Chinn S, Jarvis D et al. Determinants of cough in young adults participating in the European Community Respiratory Health Survey. Eur Respir J 2001; 18: 647-654

30 Kelsall A, Decalmer S, McGuiness $K$ et al. Sex differences and predictors of objective cough frequency in chronic cough. Thorax 2009; 64: $393-$ 398

31 Kelsall A, Houghton LA, Jones $H$ et al. A novel approach to studying the relationship between subjective and objective measures of cough. Chest 2011; 139: 569-575

32 Reinke C, Dette F, Gross V et al. Obscure coughing and the feeling of suffocation during sleep. Long-term nocturnal acoustic recording. Internist 2007; 48: 630-635

33 Sundar KM, Daly SE. A longitudinal study of CPAP therapy for patients with chronic cough and obstructive sleep apnea. Cough 2013; 9: 1925

34 Yokohori N, Hasegawa M, Sato A et al. Utility of Continuous Positive Airway Pressure Therapy for Treating Chronic Coughs in Patients with Obstructive Sleep Apnea. Intern Med 2014; 53: 1079-1082 